\title{
New Generalized Extreme Value Distribution and its Bivariate Extension
}

\author{
A. H. El-Bassiouny \\ Mansoura University \\ Mathematics Department \\ Faculty of Science
}

\author{
Mohamed Abouhawwash \\ Mansoura University \\ Mathematics Department \\ Faculty of Science
}

\author{
H. S. Shahen \\ Mansoura University \\ Mathematics Department \\ Faculty of Science
}

\begin{abstract}
In this paper, a new distribution called New Generalized Extreme Value (NGEV) distribution is introduced. Also, the statistical properties of this model are studied, such as, quantiles, moment generating function and moments of order statistics. Moreover, maximum likelihood estimators of it's parameters are discussed. An application of NGEV distribution to a survival times in months of 20 acute myeloid leukemia patients data set is provided. Also, bivariate New Generalized Extreme Value BNGEV distribution.is introduced a Marshall-Olkin type. Marginal and conditional distribution functions are studied. Furthermore, maximum likelihood estimates (MLEs) of the parameters are presented. An application of BNGEV distribution to an UEFA Champion's League data set is provided and the profiles of the log-likelihood function of parameters of NGEVD and BNGEVD are plotted.
\end{abstract}

\section{General Terms}

Univariate distribution, Bivariate distribution

\section{Keywords}

Extreme value distribution, Exponentiated Weibull distribution, Moment generating function, Joint cumulative distribution function, Maximum likelihood estimation

\section{INTRODUCTION}

Extreme value distributions are the limiting distributions for the minimum or the maximum of a very large collection of random observations from the same arbitrary distribution. In the context of reliability modeling, extreme value distributions for the minimum are frequently encountered. The weibull distribution and the extreme value distribution have a useful mathematical relationship, i.e. the natural log of a weibull random time is an extreme value random observation.

The aim of this paper is to introduce a new generalized extreme value (NGEV) distribution by using the mathematical relationship between weibull distribution and extreme value distribution. Also, we introduce its bivariate and we call it bivariate New Generalized Extreme Value (BNGEV) distribution, whose marginals are NGEV distributions. It is a Marshall-Olkin type. Many authors used this method to introduce a new bivariate distributions, see for example Marshall and Olkin [2], Kundu and Gupta [1], Sarhan and Balakr- ishnan [3], Sarhan et al. [6], El-Bassiouny et al.[4] and El-Gohary et al. [5].

This article is organized as follows, the new generalized extreme value (NGEV) distribution are proposed in Section 2. Various properties including quantiles, median and moment generating function are investigated in Section 3. Rényi entropy is provided in Section 4. Moments of order statistics are obtained in Section 4. Section 5 is devoted to the maximum likelihood estimates of the parameters and the information matrix of the NGEV distribution. An application of NGEV distribution to a survival times in months of 20 acute myeloid leukemia patients data set is provided and the profiles of the log-likelihood function of parameters of NGED are ploted in section 6 . In section 7 , we introduced the bivariate case and we call it bivariate New Generalized Extreme Value (BNGEV) distribution. Also, various properties including the joint survival function, the joint cumulative distribution function, the joint probability density function, marginal probability density functions are investigated in Section 8. Section 9 is devoted to the maximum likelihood estimates of the parameters of the BNGEV distribution. In Section 10, an application of the BNGE distribution to a UEFA Champion's League data set are provided and the profiles of the log-likelihood function of parameters of BNGED are ploted. Finally, the results of this paper are concluded in Section 11.

\section{NEW GENERALIZED EXTREME VALUE DISTRIBUTION}

In this section, we discuss the new generalized extreme value (NGEV) distribution. This distribution is derived from an exponentiated weibull distribution. Let $X$ be a random variable has Exponentiated Weibull (EW) distribution [9] with parameters $\alpha, \lambda$ and $k>0$, then its cdf is given by

$$
G(x)=\left(1-e^{-\left(\frac{x}{\lambda}\right)^{k}}\right)^{\alpha}, x \geq 0, \alpha, k, \lambda>0
$$

and the probability density function (pdf) is given by

$$
g(x)=\frac{\alpha k}{\lambda}\left(\frac{x}{\lambda}\right)^{k-1} e^{-\left(\frac{x}{\lambda}\right)^{k}}\left(1-e^{-\left(\frac{x}{\lambda}\right)^{k}}\right)^{\alpha-1}, x \geq 0, \alpha, k, \lambda>0
$$




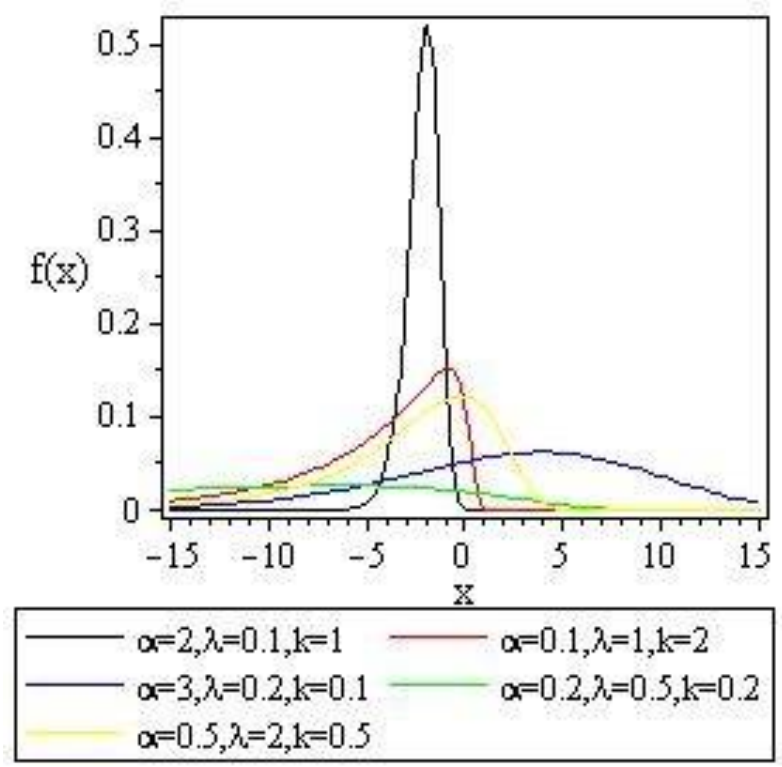

Fig. 1. The pdf of the NGEV distribution at different values of its parameters.

\subsection{NGEV Specifications}

THEOREM 1. Let a non-negative random variable $Y$ has the exponentiated weibull distribution, symbolically we write $Y \sim$ $E W(\alpha, \lambda, k)$. Define a new random variable $X=\log Y$, then the random variable $X$ has the new generalized extreme value distribution, symbolically we write $X \sim \operatorname{NGEV}(\alpha, \lambda, k)$. The cumulative distribution function and the probability density function of $X$ are respectively given by

$$
F_{X}(x)=\left(1-e^{-\left(\frac{e^{x}}{\lambda}\right)^{k}}\right)^{\alpha},-\infty<x<\infty, \alpha, k, \lambda>0
$$

and

$$
f_{X}(x)=\alpha k\left(\frac{e^{x}}{\lambda}\right)^{k} e^{-\left(\frac{e^{x}}{\lambda}\right)^{k}}\left(1-e^{-\left(\frac{e^{x}}{\lambda}\right)^{k}}\right)^{\alpha-1} .
$$

ProOF. Since

$$
\begin{aligned}
F_{X}(x) & =P[X \leq x]=P[\log Y \leq x] \\
& =P\left[Y \leq e^{x}\right]=G\left(e^{x}\right) \\
& =\left(1-e^{-\left(\frac{e^{x}}{\lambda}\right)^{k}}\right)^{\alpha},-\infty<x<\infty, \alpha, k, \lambda>0 .
\end{aligned}
$$

By diffrentiation the cdf of $X$ given in (2) with respect to $x$, we find the pdf of $X$ given in (3), which complete the proof.

Since the cdf of NGEV is in closed form, we can use it to generate simulated data by using the following formula

$$
x=\ln \left(\lambda\left(-\ln \left(1-U^{\frac{1}{\alpha}}\right)\right)^{\frac{1}{k}}\right),
$$

where $U$ is a random variable which follows a standard uniform distribution on $(0,1)$ interval.

Figures 1 and 2 illustrate some of the possible shapes of the pdf and cdf of the NGEV distribution.

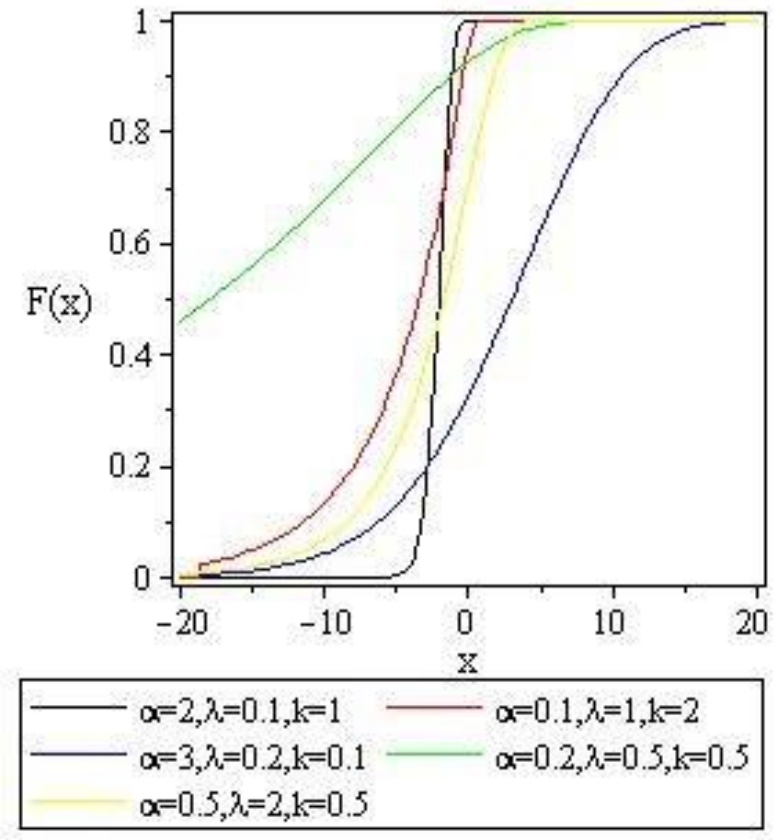

Fig. 2. The cdf of the NGEV distribution at different values of its parameters.

\section{PROPERTIES OF THE NGEV DISTRIBUTION}

In this section, we discuss some properties of the NGEV distribution.

\subsection{Quantile, Median and Mode}

The $q$ th quantile, can be computed to the NGEV distribution as

$$
x_{q}=\ln \left(\lambda\left(-\ln \left(1-q^{\frac{1}{\alpha}}\right)\right)^{\frac{1}{k}}\right), 0<q<1 .
$$

The median of the NGEV distribution, i.e. $x_{0.5}$, is given by

$$
\operatorname{median}(X)=x_{0.5}=\ln \left(\lambda\left(-\ln \left(1-(0.5)^{\frac{1}{\alpha}}\right)\right)^{\frac{1}{k}}\right) .
$$

Moreover, the mode of the NGEV distribution can be obtained by deriving its pdf with respect to $x$ and equal it to zero. Thus the mode of the NGEV distribution can be obtained as a solution of the following nonlinear equation

$$
(\alpha-1)\left(\frac{e^{x}}{\lambda}\right)^{k} e^{-\left(\frac{e^{x}}{\lambda}\right)^{k}}\left(1-e^{-\left(\frac{e^{x}}{\lambda}\right)^{k}}\right)^{-1}-\left(\frac{e^{x}}{\lambda}\right)^{k}+1=0 .
$$

One can't get an explicit solution of (6) in the general case. Numerical methods should be used to solve it.

\subsection{Moment generating function of NGEVD}

In this subsection, the moment generating function and the $r$ th moment about the origin of the NGEV distribution are computed. 
THEOREM 2. The moment generating function of this NGEV distribution is as follows

$$
M_{X}(t)=\alpha \sum_{i=0}^{\infty}\left(\begin{array}{c}
\alpha-1 \\
i
\end{array}\right)(-1)^{i} \lambda^{t} \frac{1}{(i+1)^{\frac{t}{k}+1}} \Gamma\left(\frac{t}{k}+1\right)
$$

Proof.

$$
\begin{aligned}
M_{X}(t)= & E\left(e^{t X}\right)=\int_{-\infty}^{\infty} e^{t x} f(x) d x \\
= & \int_{-\infty}^{\infty} e^{t x} \alpha k\left(\frac{e^{x}}{\lambda}\right)^{k} e^{-\left(\frac{e^{x}}{\lambda}\right)^{k}}\left(1-e^{-\left(\frac{e^{x}}{\lambda}\right)^{k}}\right)^{\alpha-1} d x \\
= & \alpha k \sum_{i=0}^{\infty}\left(\begin{array}{c}
\alpha-1 \\
i
\end{array}\right)(-1)^{i} \int_{-\infty}^{\infty} e^{t x}\left(\frac{e^{x}}{\lambda}\right)^{k} \\
& \times e^{-(i+1)\left(\frac{e^{x}}{\lambda}\right)^{k}} d x
\end{aligned}
$$

let $(i+1)\left(\frac{e^{x}}{\lambda}\right)^{k}=u, x=\ln \left(\frac{\lambda}{(i+1)^{1 / k}}\right)+\frac{1}{k} \ln (u), d x=$ $\frac{1}{k u} d u, u=0 \rightarrow \infty$

$$
\begin{aligned}
M_{X}(t) & =\alpha \sum_{i=0}^{\infty}\left(\begin{array}{c}
\alpha-1 \\
i
\end{array}\right)(-1)^{i} \lambda^{t} \frac{1}{(i+1)^{\frac{t}{k}+1}} \int_{0}^{\infty} e^{-u} u^{\frac{t}{k}} d u \\
& =\alpha \sum_{i=0}^{\infty}\left(\begin{array}{c}
\alpha-1 \\
i
\end{array}\right)(-1)^{i} \lambda^{t} \frac{1}{(i+1)^{\frac{t}{k}+1}} \Gamma\left(\frac{t}{k}+1\right) .
\end{aligned}
$$

The $r t h$ moment about the origin of the NGEV distribution is given by $E\left(X^{r}\right)=\left.\frac{d^{r}}{d t^{r}} M_{X}(t)\right|_{t=0}$ and by using maple software, we find $M_{X}(0)=1$.

\section{RÉNYI ENTROPY OF NGEVD}

In this section, we compute the rényi entropy which is a measure of the uncertain variation

THEOREM 3. The Rényi entropies for the NGEV distribution are given by

$$
I_{R}(\delta)=\frac{1}{1-\delta} \log [I(\delta)], \delta \neq 1
$$

where

$$
I(\delta)=\alpha^{\delta} k^{\delta-1} \sum_{i=0}^{\infty}\left(\begin{array}{c}
\alpha \delta-\delta \\
i
\end{array}\right)(-1)^{i}\left(\frac{1}{\delta+i}\right)^{\delta} \Gamma(\delta)
$$

ProOF.

$$
\begin{gathered}
I_{R}(\delta)=\frac{1}{1-\delta} \log (I(\delta)) \\
I(\delta)=\int_{\Re} f^{\delta}(x) d x=\int_{-\infty}^{\infty} \alpha^{\delta} k^{\delta}\left(\frac{e^{x}}{\lambda}\right)^{\delta k} e^{-\delta\left(\frac{e^{x}}{\lambda}\right)^{k}} \\
\times\left(1-e^{-\left(\frac{e^{x}}{\lambda}\right)^{k}}\right)^{\alpha \delta-\delta} d x \\
=\alpha^{\delta} k^{\delta} \sum_{i=0}^{\infty}\left(\begin{array}{c}
\alpha \delta-\delta \\
i
\end{array}\right)(-1)^{i} \int_{-\infty}^{\infty}\left(\frac{e^{x}}{\lambda}\right)^{\delta k} e^{-(\delta+i)\left(\frac{e^{x}}{\lambda}\right)^{k}} d x
\end{gathered}
$$

let $(\delta+i)\left(\frac{e^{x}}{\lambda}\right)^{k}=u, x=\ln \left(\frac{\lambda}{(\delta+i)^{1 / k}}\right)+\frac{1}{k} \ln (u), d x=$ $\frac{1}{k u} d u, u=0 \rightarrow \infty$

$$
\begin{aligned}
I(\delta) & =\alpha^{\delta} k^{\delta-1} \sum_{i=0}^{\infty}\left(\begin{array}{c}
\alpha \delta-\delta \\
i
\end{array}\right)(-1)^{i}\left(\frac{1}{\delta+i}\right)^{\delta} \int_{0}^{\infty} e^{-u} u^{\delta-1} d u \\
& =\alpha^{\delta} k^{\delta-1} \sum_{i=0}^{\infty}\left(\begin{array}{c}
\alpha \delta-\delta \\
i
\end{array}\right)(-1)^{i}\left(\frac{1}{\delta+i}\right)^{\delta} \Gamma(\delta)
\end{aligned}
$$

\section{MOMENTS OF ORDER STATISTICS OF NGEVD}

Suppose that $n$ random variables $X_{1}, X_{2}, \ldots, X_{n}$ are ordered in non-decreasing magnitude and written as $X_{1: n} \leq X_{2: n} \leq \ldots \leq$ $X_{n: n}$. Where the smallest order statistic is denoted by $X_{1: n}$, the second smallest is denoted by $X_{2: n}$, and so on, and the largest order statistic is denoted by $X_{n: n}$ and $X_{r: n}$ is called $r^{t h}$ order statistic. In the definition of order statistics, there is no restriction on whether $X_{r} \mathrm{~s}$ are independent or identically distributed. But many wellknown results about order statistics are under the classical assumption that $X_{r}$ s are independent and identically distributed (iid). The $p d f$ of the $r^{\text {th }}$ order statistic is

$$
\begin{aligned}
f_{r: n}(x) & =\frac{n !}{(n-r) !(r-1) !} f(x)[F(x)]^{r-1}[1-F(x)]^{n-r} \\
,-\infty & <x<\infty
\end{aligned}
$$

where $f(x)$ comes from 3 and $F(x)$ comes from 2 .

The $k$ th moment about zero of the $r$ th order statistic is given by the following theorem

THEOREM 4. The kth moment about zero of the rth order statistic for the NGEV distribution is given by

$$
\begin{aligned}
\mu_{r: n}^{(k)}= & \frac{n !}{(r-1) !(n-r) !} \alpha \sum_{i=0}^{n-r} \sum_{j=0}^{\infty}\left(\begin{array}{c}
n-r \\
i
\end{array}\right)\left(\begin{array}{c}
\alpha(r+i)-1 \\
j
\end{array}\right) \\
& \times(-1)^{i+j} \frac{1}{j+1}
\end{aligned}
$$

PROOF.

$$
\begin{aligned}
\mu_{r: n}^{(k)}= & \frac{n !}{(r-1) !(n-r) !} \int_{-\infty}^{\infty} f(x)[F(x)]^{r-1}(1-F(x))^{n-r} d x \\
= & \frac{n !}{(r-1) !(n-r) !} \alpha k \sum_{i=0}^{n-r}\left(\begin{array}{c}
n-r \\
i
\end{array}\right)(-1)^{i} \int_{-\infty}^{\infty}\left(\frac{e^{x}}{\lambda}\right)^{k} \\
& \times e^{-\left(\frac{e^{x}}{\lambda}\right)^{k}}\left(1-e^{-\left(\frac{e^{x}}{\lambda}\right)^{k}}\right)^{\alpha(r+i)-1} d x \\
= & \frac{n !}{(r-1) !(n-r) !} \alpha k \sum_{i=0}^{n-r} \sum_{j=0}^{\infty}\left(\begin{array}{c}
n-r \\
i
\end{array}\right)\left(\begin{array}{c}
\alpha(r+i)-1 \\
j
\end{array}\right) \\
& \times(-1)^{i+j} \int_{-\infty}^{\infty}\left(\frac{e^{x}}{\lambda}\right)^{k} e^{-(j+1)\left(\frac{e^{x}}{\lambda}\right)^{k}} d x
\end{aligned}
$$


let $(j+1)\left(\frac{e^{x}}{\lambda}\right)^{k}=u, x=\ln \left(\frac{\lambda}{(i+1)^{1 / k}}\right)+\frac{1}{k} \ln (u), d x=$ $\frac{1}{k u} d u, u=0 \rightarrow \infty$

$$
\begin{aligned}
\mu_{r: n}^{(k)}= & \frac{n !}{(r-1) !(n-r) !} \alpha \sum_{i=0}^{n-r} \sum_{j=0}^{\infty}\left(\begin{array}{c}
n-r \\
i
\end{array}\right)\left(\begin{array}{c}
\alpha(r+i)-1 \\
j
\end{array}\right) \\
& \times \frac{(-1)^{i+j}}{j+1} \int_{0}^{\infty} e^{-u} d u \\
= & \frac{n !}{(r-1) !(n-r) !} \alpha \sum_{i=0}^{n-r} \sum_{j=0}^{\infty}\left(\begin{array}{c}
n-r \\
i
\end{array}\right)\left(\begin{array}{c}
\alpha(r+i)-1 \\
j
\end{array}\right) \\
& \times(-1)^{i+j} \frac{1}{j+1}
\end{aligned}
$$

\section{ESTIMATION AND INFORMATION MATRIX OF NGEVD}

In this section we discussed the estimation of the NGEV parameters by using the method of maximum likelihood based on a complete sample.

\subsection{Maximum Likelihood Estimators}

Let $x_{1}, x_{2}, \ldots, x_{n}$ be a random sample from NGEV distribution. Then the log-likelihood function of the NGEV may be expressed as

$$
\begin{aligned}
L= & \log l(x ; \alpha, k, \lambda)=n \log \alpha+n \log k+k \sum_{i=1}^{n} \log \left(\frac{e^{x_{i}}}{\lambda}\right) \\
& -\sum_{i=1}^{n}\left(\frac{e^{x_{i}}}{\lambda}\right)^{k}+(\alpha-1) \sum_{i=1}^{n} \log \left(1-e^{-\left(\frac{e^{x_{i}}}{\lambda}\right)^{k}}\right)
\end{aligned}
$$

Differentiating the log-likelihood with respect to $\alpha, \lambda$ and $k$, respectively, and setting the result equal to zero, we have

$$
\begin{aligned}
\frac{\partial L}{\partial \alpha}= & \frac{n}{\alpha}+\sum_{i=1}^{n} \log \left(1-e^{-\left(\frac{e^{x_{i}}}{\lambda}\right)^{k}}\right) \\
\frac{\partial L}{\partial k}= & \frac{n}{k}+\sum_{i=1}^{n} \log \left(\frac{e^{x_{i}}}{\lambda}\right)-\sum_{i=1}^{n}\left(\frac{e^{x_{i}}}{\lambda}\right)^{k} \log \left(\frac{e^{x_{i}}}{\lambda}\right) \\
& +(\alpha-1) \sum_{i=1}^{n} \frac{e^{-\left(\frac{e^{x_{i}}}{\lambda}\right)^{k}}\left(\frac{e^{x_{i}}}{\lambda}\right)^{k} \log \left(\frac{e^{x_{i}}}{\lambda}\right)}{1-e^{-\left(\frac{e^{x_{i}}}{\lambda}\right)^{k}}} \\
\frac{\partial L}{\partial \lambda}= & \frac{-n k}{\lambda}+\frac{k}{\lambda} \sum_{i=1}^{n}\left(\frac{e^{x_{i}}}{\lambda}\right)^{k}-\frac{(\alpha-1) k}{\lambda} \\
& \times \sum_{i=1}^{n} \frac{e^{-\left(\frac{e^{x_{i}}}{\lambda}\right)^{k}}\left(\frac{e^{x_{i}}}{\lambda}\right)^{k}}{1-e^{-\left(\frac{e^{x_{i}}}{\lambda}\right)^{k}}}
\end{aligned}
$$

The maximum likelihood estimates $\widehat{\alpha}, \widehat{\lambda}$ and $\widehat{k}$ of the unknown parameters $\alpha, \lambda$ and $k$ respectively, are obtained by setting these above equations $[10)-(12)$ to zero and solving them simultaneously.

\subsection{Asymptotic Confidence Bounds}

In this subsection, we derive the asymptotic confidence intervals of the unknown parameters $\alpha, \lambda$ and $k$ when $\alpha, \lambda>0$ and $k>0$. The simplest large sample approach is to assume that the $\operatorname{MLEs}(\alpha, \lambda, k)$ are approximately multivariate normal with mean $(\alpha, \lambda, k)$ and covariance matrix $I_{0}^{-1}$ where $I_{0}^{-1}$ the inverse of the observed information matrix which is defined by

$$
\begin{gathered}
I_{0}^{-1}=-\left[\begin{array}{ccc}
\frac{\partial^{2} L}{\partial \alpha^{2}} & \frac{\partial^{2} L}{\partial \alpha \partial \lambda} & \frac{\partial^{2} L}{\partial \alpha \partial k} \\
\frac{\partial^{2} L}{\partial \lambda \partial \alpha} & \frac{\partial^{2} L}{\partial \lambda^{2}} & \frac{\partial^{2} L}{\partial \lambda \partial k} \\
\frac{\partial^{2} L}{\partial k \partial \alpha} & \frac{\partial^{2} L}{\partial k \partial \lambda} & \frac{\partial^{2} L}{\partial k^{2}}
\end{array}\right]^{-1} \\
=\left[\begin{array}{ccc}
\operatorname{var}(\widehat{\alpha}) & \operatorname{cov}(\widehat{\alpha}, \widehat{\lambda}) & \operatorname{cov}(\widehat{\alpha}, \widehat{k}) \\
\operatorname{cov}(\widehat{\lambda}, \widehat{\alpha}) & \operatorname{var}(\widehat{\lambda}) & \operatorname{cov}(\widehat{\lambda}, \widehat{k}) \\
\operatorname{cov}(\widehat{k}, \widehat{\alpha}) & \operatorname{cov}(\widehat{k}, \widehat{\lambda}) & \operatorname{var}(\widehat{k})
\end{array}\right]
\end{gathered}
$$

The second partial derivatives included in $I_{0}^{-1}$ are given as follows $\frac{\partial^{2} L}{\partial \alpha^{2}}=\frac{-n}{\alpha^{2}}$

$$
\begin{aligned}
& \frac{\partial^{2} L}{\partial k^{2}} \quad=\quad \frac{-n}{k^{2}}-\sum_{i=1}^{n}\left(\frac{e^{x_{i}}}{\lambda}\right)^{k}\left(\log \left(\frac{e^{x_{i}}}{\lambda}\right)\right)^{2}+ \\
& (\alpha-1) \sum_{i=1}^{n} \frac{\left(\log \left(\frac{e^{x_{i}}}{\lambda}\right)\right) W_{i}\left(1-e^{\left.-\left(\frac{e^{x_{i}}}{\lambda}\right)^{k}\right)}\right)\left(1-\left(\frac{e^{x_{i}}}{\lambda}\right)^{k}\right)-W_{i}^{2}}{\left(1-e^{-\left(\frac{e^{x_{i}}}{\lambda}\right)^{k}}\right)^{2}} \\
& \frac{\partial^{2} L}{\partial \lambda^{2}}=\frac{n k}{\lambda^{2}}-\frac{k(1-k)}{\lambda^{2}} \sum_{i=1}^{n}\left(\frac{e^{x_{i}}}{\lambda}\right)^{k}+\frac{(\alpha-1) k}{\lambda^{2}} \sum_{i=1}^{n} \frac{R_{i}}{1-e^{-\left(\frac{e^{x_{i}}}{\lambda}\right)^{k}}-} \\
& \frac{(\alpha-1) k}{\lambda} \sum_{i=1}^{n} \frac{\left(1-e^{-\left(\frac{e^{x_{i}}}{\lambda}\right)^{k}}\right) \frac{k}{\lambda} R_{i}\left(\left(\frac{e^{x_{i}}}{\lambda}\right)^{k}-1\right)+\frac{k}{\lambda} R_{i}^{2}}{\left(1-e^{-\left(\frac{e^{x_{i}}}{\lambda}\right)^{k}}\right)^{2}} \\
& \frac{\partial^{2} L}{\partial \alpha \partial k}=\sum_{i=1}^{n} \frac{W_{i}}{1-e^{-\left(\frac{e^{x_{i}}}{\lambda}\right)^{k}}} \\
& \frac{\partial^{2} L}{\partial \alpha \partial \lambda}=\sum_{i=1}^{n} \frac{\frac{-k}{\lambda} R_{i}}{1-e^{-\left(\frac{e^{x_{i}}}{\lambda}\right)^{k}}} \\
& \frac{\partial^{2} L}{\partial k \partial \lambda}=\frac{-n}{\lambda}+\frac{1}{\lambda} \sum_{i=1}^{n}\left(\frac{e^{x_{i}}}{\lambda}\right)^{k}+\frac{k}{\lambda} \sum_{i=1}^{n}\left(\frac{e^{x_{i}}}{\lambda}\right)^{k} \log \left(\frac{e^{x_{i}}}{\lambda}\right)- \\
& \frac{(\alpha-1) k}{\lambda} \sum_{i=1}^{n} \frac{\left(1-e^{-\left(\frac{e^{x_{i}}}{\lambda}\right)^{k}}\right) W_{i}\left[-\left(\frac{e^{x_{i}}}{\lambda}\right)^{k}+1\right]-R_{i}^{2} \log \left(\frac{e^{x_{i}}}{\lambda}\right)}{\left(1-e^{-\left(\frac{e^{x_{i}}}{\lambda}\right)^{k}}\right)^{2}} \\
& \frac{(\alpha-1)}{\lambda} \sum_{i=1}^{n} \frac{R_{i}}{1-e^{-\left(\frac{e^{x_{i}}}{\lambda}\right)^{k}}}
\end{aligned}
$$

where the functions $W_{i}$ and $R_{i}$ are given by

$W_{i}=\log \left(\frac{e^{x_{i}}}{\lambda}\right) e^{-\left(\frac{e^{x_{i}}}{\lambda}\right)^{k}}\left(\frac{e^{x_{i}}}{\lambda}\right)^{k}$,

$R_{i}=e^{-\left(\frac{e^{x_{i}}}{\lambda}\right)^{k}}\left(\frac{e^{x_{i}}}{\lambda}\right)^{k}$.

The above approach is used to derive the $(1-\delta) 100 \%$ confidence intervals for the parameters $\alpha, \lambda$ and $k$ as in the following forms

$$
\widehat{\alpha} \pm Z_{\frac{\delta}{2}} \sqrt{\operatorname{var}(\widehat{\alpha})}, \widehat{\lambda} \pm Z_{\frac{\delta}{2}} \sqrt{\operatorname{var}(\widehat{\lambda})}, \widehat{k} \pm Z_{\frac{\delta}{2}} \sqrt{\operatorname{var}(\widehat{k})},
$$

where $Z_{\frac{\delta}{2}}$ is the upper $\left(\frac{\delta}{2}\right) t h$ percentile of the standard normal distribution. 


\section{APPLICATION OF THE NGEV DISTRIBUTION}

Now we use a real data set to show that the NGEV distribution is competitive among other known distributions such as Gumbel type2 (G type-2) distribution, Exponentiated Fréchet (EF) distribution, Fréchet distribution and lognormal (LN) distribution. The data set in Table 1 shows the survival times in months of 20 acute myeloid leukemia patients reported in Afify et al. [19].

Table1: Survival times in months of 20 acute myeloid leukemia patients

\begin{tabular}{lllllll}
\hline \hline 2.226 & 2.113 & 3.631 & 2.473 & 2.720 & 2.746 & 1.972 \\
\hline \hline 2.050 & 2.061 & 3.915 & 0.871 & 1.548 & 2.808 & 1.079 \\
\hline \hline 1.200 & 0.726 & 2.967 & 1.958 & 2.265 & 2.353 & \\
\hline \hline
\end{tabular}

The MLEs of the unknown parameters $\alpha, \lambda$ and $k$ are given in Table 2. Also, the values of the log-likelihood functions $L$, the statistics $A I C$ (Akaike Information Criterion), $C A I C$ (Consistent AkaikeInformation Criteria) and $B I C$ (Bayesian Information Criterion) are given in Table 3 for the five distributions in order to verify which distribution fits better this real data set.

\begin{tabular}{c||c|c|}
\multicolumn{4}{c}{ Table 2: The MLEs and the values of $L$} \\
\hline \hline Model & MLEs & $L$ \\
\hline \hline G type-2 & $\widehat{\theta}=2.6040, \widehat{\phi}=2.0651$ & -29.08667 \\
\hline EF & $\widehat{\alpha}=0.3928, \widehat{\phi}=3.4393$ & -31.89448 \\
\hline Fréchet & $\widehat{\phi}=1.7378$ & -35.45610 \\
\hline LN & $\widehat{\mu}=0.6971, \widehat{\sigma}=0.4360$ & -25.71549 \\
\hline NGEV & $\widehat{\alpha}=26.389, \widehat{\lambda}=0.292$ & -24.347 \\
& $\widehat{k}=0.382$ & \\
\hline
\end{tabular}

\begin{tabular}{|c|c|c|c|}
\hline Model & $\overline{A I C}$ & $\overline{B I C}$ & $C A I C$ \\
\hline G type-2 & 62.1733 & 64.1648 & 66.1648 \\
\hline $\mathrm{EF}$ & 67.7889 & 69.7804 & 71.7804 \\
\hline Fréchet & 72.9122 & 76.9037 & 74.9079 \\
\hline $\mathrm{LN}$ & 55.431 & 57.4225 & 59.4225 \\
\hline NGEV & 54.694 & 52.5971 & 56.194 \\
\hline
\end{tabular}

Since the values of $-L, A I C, B I C$ and $C A I C$ (see Table 2 and Table 3) are smaller for the NGEV distribution compared with those values of the other models, then the new distribution seems to be a very competitive model based on this real data set.

Substituting the MLEs of the unknown parameters into (13), we get an estimation of the variance covariance matrix as the following:

$$
I_{0}^{-1}=\left[\begin{array}{ccc}
1.559 \times 10^{3} & -27.535 & -5.764 \\
-27.535 & 0.513 & 0.111 \\
-5.764 & 0.111 & 0.025
\end{array}\right]
$$

The approximate 95\% two sided confidence intervals of the unknown parameters $\alpha, \lambda$ and $k$ are, respectively,given as [50.99,103.78], [-1.112,1.696], [0.0721,0.6919].

The profiles of the log-likelihood functions of $\alpha, \lambda$ and $k$ of NGEV for survival times in months of 20 acute myeloid leukemia patients data are ploted in Fig. 3 and Fig. 4. From the plots of the profiles of the log-likelihood function of $\alpha, \lambda$ and $k$, we observe that the likelihood equations have a unique solution.

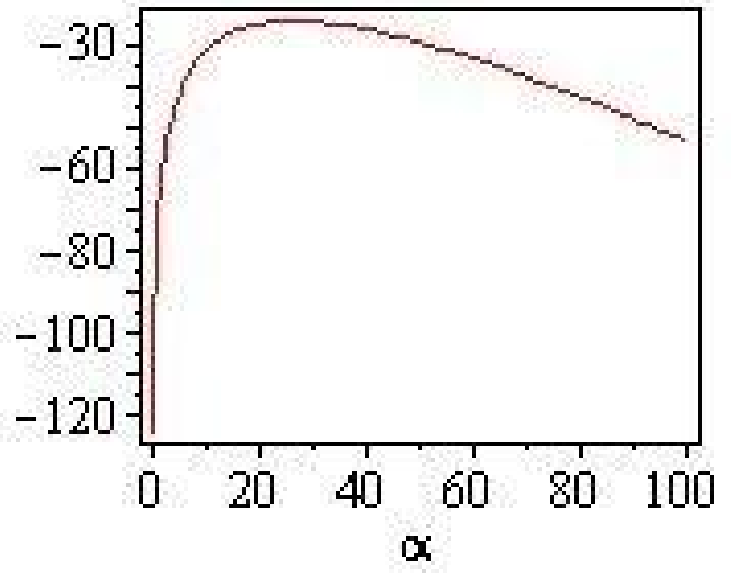

Fig. 3. The profile of the log-likelihood function of $\alpha$.

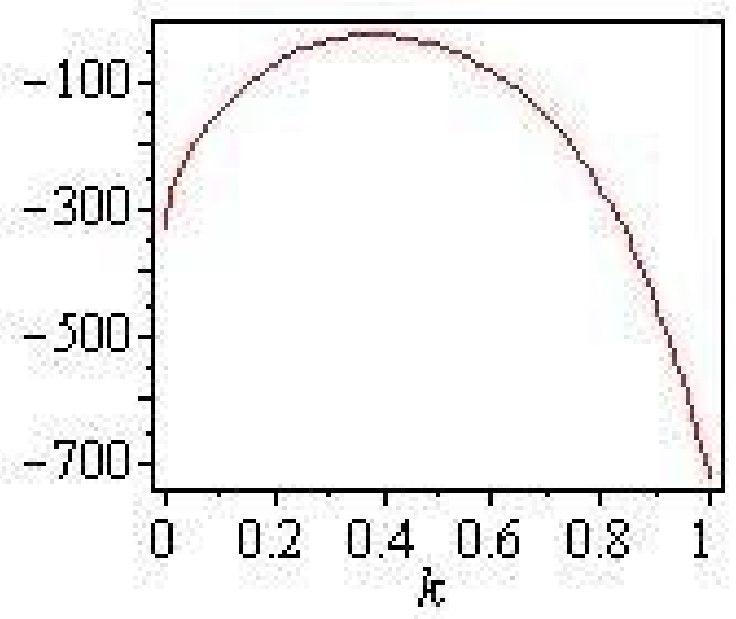

Fig. 4. The profile of the log-likelihood function of k.

\section{BIVARIATE NEW GENERALIZED EXTREME VALUE DISTRIBUTION}

In this section, we discuss the BNGEV distribution.We start with the joint survival function and derive the corresponding joint probability density function of this distribution

\subsection{The Joint Cumulative Distribution Function}

Suppose that $U_{1} \sim N G E V\left(\alpha_{1}, \lambda, k\right), U_{2} \sim N G E V\left(\alpha_{2}, \lambda, k\right)$ and $U_{3} \sim N G E V\left(\alpha_{3}, \lambda, k\right)$ are independent random variables. Define $X_{1}=\max \left\{U_{1}, U_{3}\right\}$ and $X_{2}=\max \left\{U_{2}, U_{3}\right\}$. Then, the bivariate vector $\left(X_{1}, X_{2}\right) \sim B N G E V\left(\alpha_{1}, \alpha_{2}, \alpha_{3}, \lambda, k\right)$ In the following lemma, We study the joint cumulative distribution function of the random varibles $X_{1}$ and $X_{2}$.

LEMMA 5. The joint $c d f$ of $X_{1}$ and $X_{2}$ is given by 


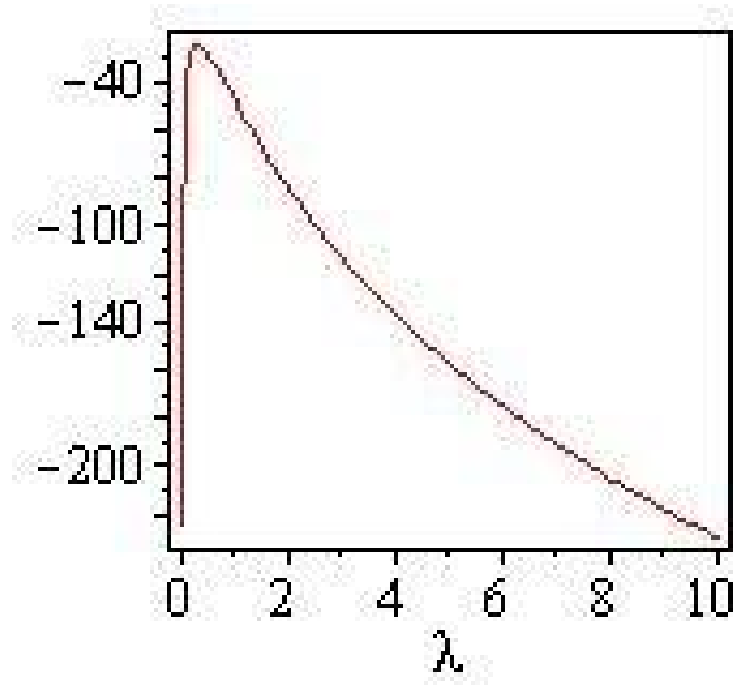

Fig. 5. The profile of the log-likelihood function of $\lambda$.

$$
\begin{aligned}
F_{\text {BNGEV }}\left(x_{1}, x_{2}\right)= & \left(1-e^{-\left(\frac{e^{x_{1}}}{\lambda}\right)^{k}}\right)^{\alpha_{1}}\left(1-e^{-\left(\frac{e^{x_{2}}}{\lambda}\right)^{k}}\right)^{\alpha_{2}} \\
& \times\left(1-e^{-\left(\frac{e^{z}}{\lambda}\right)^{k}}\right)^{\alpha_{3}}
\end{aligned}
$$

where $z=\min \left(x_{1}, x_{2}\right)$.

ProOF.

$$
\begin{aligned}
F_{B N G E V}\left(x_{1}, x_{2}\right)= & P\left(X_{1} \leq x_{1}, X_{2} \leq x_{2}\right) \\
= & P\left(\max \left\{U_{1}, U_{3}\right\} \leq x_{1},\right. \\
& \left.\max \left\{U_{2}, U_{3}\right\} \leq x_{2}\right) \\
= & P\left(U_{1} \leq x_{1}, U_{2} \leq x_{2},\right. \\
& \left.U_{3} \leq \min \left(x_{1}, x_{2}\right)\right) .
\end{aligned}
$$

Where, $U_{i}(i=1,2,3)$ are mutually independent random variables. Then, we obtain

$$
\begin{aligned}
F_{B N G E V}\left(x_{1}, x_{2}\right)= & P\left(U_{1} \leq x_{1}\right) P\left(U_{2} \leq x_{2}\right) \\
& \times P\left(U_{3} \leq \min \left(x_{1}, x_{2}\right)\right) \\
= & F_{N G E V}\left(x_{1} ; \alpha_{1}, \lambda, k\right) F_{N G E V}\left(x_{2} ; \alpha_{2}, \lambda, k\right) \\
& \times F_{N G E V}\left(z ; \alpha_{3}, \lambda, k\right) \\
= & \left(1-e^{-\left(\frac{e^{x_{1}}}{\lambda}\right)^{k}}\right)^{\alpha_{1}}\left(1-e^{-\left(\frac{e^{x_{2}}}{\lambda}\right)^{k}}\right)^{\alpha_{2}} \\
& \times\left(1-e^{-\left(\frac{e^{z}}{\lambda}\right)^{k}}\right)^{\alpha_{3}} .
\end{aligned}
$$

\subsection{The Joint Probability Density Function}

In this subsection, we study the joint probability density function of the random varibles $X_{1}$ and $X_{2}$ in the following theorem.
THEOREM 6. If the joint cdf of $\left(X_{1}, X_{2}\right)$ is as in 14 then, the joint $p d f$ of $\left(X_{1}, X_{2}\right)$ is given by

$$
f_{B N G E V}\left(x_{1}, x_{2}\right)=\left\{\begin{array}{l}
f_{1}\left(x_{1}, x_{2}\right) \text { if } x_{1}<x_{2} \\
f_{2}\left(x_{1}, x_{2}\right) \text { if } x_{2}<x_{1} \\
f_{3}(x) \text { if } x_{1}=x_{2}=x
\end{array}\right.
$$

where

$$
\begin{aligned}
f_{1}\left(x_{1}, x_{2}\right)= & f_{N G E V}\left(x_{1} ; \alpha_{1}+\alpha_{3}, \lambda, k\right) f_{N G E V}\left(x_{2} ; \alpha_{2}, \lambda, k\right) \\
= & \left(\alpha_{1}+\alpha_{3}\right) k^{2}\left(\frac{e^{x_{1}}}{\lambda}\right)^{k} e^{-\left(\frac{e^{x_{1}}}{\lambda}\right)^{k}} \alpha_{2}\left(\frac{e^{x_{2}}}{\lambda}\right)^{k} \\
& \times e^{-\left(\frac{e_{2}^{x}}{\lambda}\right)^{k}}\left(1-e^{-\left(\frac{e^{x_{1}}}{\lambda}\right)^{k}}\right)^{\alpha_{1}+\alpha_{3}-1} \\
& \times\left(1-e^{-\left(\frac{e^{x_{2}}}{\lambda}\right)^{k}}\right)^{\alpha_{2}-1}
\end{aligned}
$$

$f_{2}\left(x_{1}, x_{2}\right)=f_{N G E V}\left(x_{1} ; \alpha_{1}, \lambda, k\right) f_{N G E V}\left(x_{2} ; \alpha_{2}+\alpha_{3}, \lambda, k\right)$

$$
\begin{aligned}
= & \alpha_{1} k^{2}\left(\frac{e^{x_{1}}}{\lambda}\right)^{k} e^{-\left(\frac{e^{x_{1}}}{\lambda}\right)^{k}}\left(1-e^{-\left(\frac{e^{x_{1}}}{\lambda}\right)^{k}}\right)^{\alpha_{1}-1} \\
& \times\left(\alpha_{2}+\alpha_{3}\right)\left(\frac{e^{x_{2}}}{\lambda}\right)^{k} e^{-\left(\frac{e^{x_{2}}}{\lambda}\right)^{k}} \\
& \times\left(1-e^{-\left(\frac{e^{x_{2}}}{\lambda}\right)^{k}}\right)^{\alpha_{2}+\alpha_{3}-1}
\end{aligned}
$$

$$
\begin{aligned}
f_{3}(x) & =\frac{\alpha_{3}}{\alpha_{1}+\alpha_{2}+\alpha_{3}} f_{N G E V}\left(x ; \alpha_{1}+\alpha_{2}+\alpha_{3}, \lambda, k\right) \\
& =\alpha_{3} k\left(\frac{e^{x}}{\lambda}\right)^{k} e^{-\left(\frac{e^{x}}{\lambda}\right)^{k}}\left(1-e^{-\left(\frac{e^{x}}{\lambda}\right)^{k}}\right)^{\alpha_{1}+\alpha_{2}+\alpha_{3}-1}
\end{aligned}
$$

Proof. Let us first assume that $x_{1}<x_{2}$. Then, $F_{B N G E V}\left(x_{1}, x_{2}\right)$ in 14 becomes

$F_{B N G E V}\left(x_{1}, x_{2}\right)=\left(1-e^{-\left(\frac{e^{x_{1}}}{\lambda}\right)^{k}}\right)^{\alpha_{1}+\alpha_{3}}\left(1-e^{-\left(\frac{e^{x_{2}}}{\lambda}\right)^{k}}\right)^{\alpha_{2}}$.

Then, upon differentiating this function w.r.t. $x_{1}$ and $x_{2}$ we obtain the expression of $f_{1}\left(x_{1}, x_{2}\right)$ gives in $(15)$. By the same way we obtain $f_{2}\left(x_{1}, x_{2}\right)$ when $x_{2}<x_{1}$. But $f_{3}(x)$ cannot be derived in a similar way. For this reason, we use the following identity to derive $f_{3}(x)$

$\int_{-\infty}^{\infty} \int_{-\infty}^{x_{2}} f_{1}\left(x_{1}, x_{2}\right) d x_{1} d x_{2}+\int_{-\infty}^{\infty} \int_{-\infty}^{x_{1}} f_{2}\left(x_{1}, x_{2}\right) d x_{2} d x_{1}+$ $\int_{-\infty}^{\infty} f_{3}(x, x) d x=1$

$I_{1}=\int_{-\infty}^{\infty} \int_{-\infty}^{x_{2}} f_{1}\left(x_{1}, x_{2}\right) d x_{1} d x_{2} \quad$ and $\quad I_{2}=$ $\int_{-\infty}^{\infty} \int_{-\infty}^{x_{1}} f_{2}\left(x_{1}, x_{2}\right) d x_{2} d x_{1}$

then

$$
\begin{aligned}
& I_{1}=\int_{-\infty}^{\infty} \int_{-\infty}^{x_{2}}\left(\alpha_{1}+\alpha_{3}\right) k^{2}\left(\frac{e^{x_{1}}}{\lambda}\right)^{k}\left(1-e^{-\left(\frac{e^{x_{1}}}{\lambda}\right)^{k}}\right)^{\alpha_{1}+\alpha_{3}-1} \\
& \times \alpha_{2} e^{-\left(\frac{e^{x_{1}}}{\lambda}\right)^{k}}\left(\frac{e^{x_{2}}}{\lambda}\right)^{k} e^{-\left(\frac{e^{x_{2}}}{\lambda}\right)^{k}}\left(1-e^{-\left(\frac{e^{x_{2}}}{\lambda}\right)^{k}}\right)^{\alpha_{2}-1} d x_{1} d x_{2} \\
& =\int_{-\infty}^{\infty} \alpha_{2} k\left(\frac{e^{x_{2}}}{\lambda}\right)^{k} e^{-\left(\frac{e^{x_{2}}}{\lambda}\right)^{k}}\left(1-e^{-\left(\frac{e^{x_{2}}}{\lambda}\right)^{k}}\right)^{\alpha_{1}+\alpha_{2}+\alpha_{3}-1} d x_{2}
\end{aligned}
$$


Similarly

$$
\begin{aligned}
I_{2}= & \int_{-\infty}^{\infty} \alpha_{1} k\left(\frac{e^{x_{1}}}{\lambda}\right)^{k} e^{-\left(\frac{e^{x_{1}}}{\lambda}\right)^{k}} \\
& \times\left(1-e^{-\left(\frac{e^{x_{1}}}{\lambda}\right)^{k}}\right)^{\alpha_{1}+\alpha_{2}+\alpha_{3}-1} d x_{1}
\end{aligned}
$$

From (18) and (19), we get

$\int_{0}^{\infty} f_{3}(x) d x=1-I_{1}-I_{2}$

$=\int_{-\infty}^{\infty}\left(\alpha_{1}+\alpha_{2}+\alpha_{3}\right) k\left(\frac{e^{x}}{\lambda}\right)^{k} e^{-\left(\frac{e^{x}}{\lambda}\right)^{k}}$

$\times\left(1-e^{-\left(\frac{e^{x}}{\lambda}\right)^{k}}\right)^{\alpha_{1}+\alpha_{2}+\alpha_{3}-1} d x-$

$\int_{-\infty}^{\infty} \alpha_{2} k\left(\frac{e^{x}}{\lambda}\right)^{k} e^{-\left(\frac{e^{x}}{\lambda}\right)^{k}}\left(1-e^{-\left(\frac{e^{x}}{\lambda}\right)^{k}}\right)^{\alpha_{1}+\alpha_{2}+\alpha_{3}-1} d x-$

$\int_{-\infty}^{\infty} \alpha_{1} k\left(\frac{e^{x}}{\lambda}\right)^{k} e^{-\left(\frac{e^{x}}{\lambda}\right)^{k}}\left(1-e^{-\left(\frac{e^{x}}{\lambda}\right)^{k}}\right)^{\alpha_{1}+\alpha_{2}+\alpha_{3}-1} d x$

Then

$f_{3}(x, x)=\frac{\alpha_{3}}{\alpha_{1}+\alpha_{2}+\alpha_{3}} f_{N G E V}\left(x ; \alpha_{1}+\alpha_{2}+\alpha_{3}, \lambda, k\right)$

$=\alpha_{3} k\left(\frac{e^{x}}{\lambda}\right)^{k} e^{-\left(\frac{e^{x}}{\lambda}\right)^{k}}\left(1-e^{-\left(\frac{e^{x}}{\lambda}\right)^{k}}\right)^{\alpha_{1}+\alpha_{2}+\alpha_{3}-1}$

This completes the proof of the theorem.

\subsection{Marginal Probability Density Functions}

The following theorem gives the marginal probability density functions of $X_{1}$ and $X_{2}$.

THEOREM 7. The marginal probability density functions of $X_{i}$ $(i=1,2)$ is given by

$$
\begin{aligned}
f_{X_{i}}\left(x_{i}\right)= & \left(\alpha_{i}+\alpha_{3}\right) k\left(\frac{e^{x_{i}}}{\lambda}\right)^{k} e^{-\left(\frac{e^{x_{i}}}{\lambda}\right)^{k}} \\
& \times\left(1-e^{-\left(\frac{e^{x_{i}}}{\lambda}\right)^{k}}\right)^{\alpha_{i}+\alpha_{3}-1} \\
= & f_{N G E V}\left(x_{i} ; \alpha_{i}+\alpha_{3}, \lambda, k\right), \quad i=1,2 .
\end{aligned}
$$

PROOF. The marginal cumulative distribution function of $X_{i}$, say $F\left(x_{i}\right)$, as follows:

$$
\begin{aligned}
F\left(x_{i}\right) & =P\left(X_{i} \leq x_{i}\right) \\
& =P\left(\max \left\{U_{i}, U_{3}\right\} \leq x_{i}\right) \\
& =P\left(U_{i} \leq x_{i}, U_{3} \leq x_{i}\right)
\end{aligned}
$$

since, the random variables $U_{i}(i=1,2)$ and $U_{3}$ are mutually independent, then

$$
\begin{aligned}
F\left(x_{i}\right) & =P\left(U_{i} \leq x_{i}\right) P\left(U_{3} \leq x_{i}\right) \\
& =F_{N G E V}\left(x_{i} ; \alpha_{i}+\alpha_{3}, \lambda, k\right) \\
& =\left(1-e^{-\left(\frac{e^{x_{i}}}{\lambda}\right)^{k}}\right)^{\alpha_{i}+\alpha_{3}}
\end{aligned}
$$

Differenting w.r.t. $x_{i}$ we obtain the formula given in 20 .

\subsection{Conditional Probability Density Functions}

Given the marginal probability density functions of $X_{1}$ and $X_{2}$ we can now derive the conditional probability density functions as presented in the following theorem
THEOREM 8. The conditional probability density functions of $X_{i}$, given $X_{j}=x_{j}, f\left(x_{i} \mid x_{j}\right), i, j=1,2 ; i \neq j$, is given $b y$

$$
f_{X_{i} \mid X_{j}}\left(x_{i} \mid x_{j}\right)=\left\{\begin{array}{c}
f_{X_{i} \mid X_{j}}^{(1)}\left(x_{i} \mid x_{j}\right) \text { if } x_{j}<x_{i}, \\
f_{X_{i} \mid X_{j}}^{(2)}\left(x_{i} \mid x_{j}\right) \text { if } x_{i}<x_{j}, \\
f_{X_{i} \mid X_{j}}^{(3)}\left(x_{i} \mid x_{j}\right) \text { if } x_{i}=x_{j}=x,
\end{array}\right.
$$

where

$$
\begin{aligned}
& f_{X_{i} \mid X_{j}}^{(1)}\left(x_{i} \mid x_{j}\right)=\left(\alpha_{j}\left(\alpha_{i}+\alpha_{3}\right) k\left(\frac{e^{x_{i}}}{\lambda}\right)^{k} e^{-\left(\frac{e^{x_{i}}}{\lambda}\right)^{k}}\right. \\
& \left.\left(1-e^{-\left(\frac{e^{x_{i}}}{\lambda}\right)^{k}}\right)^{\alpha_{i}+\alpha_{3}-1}\right) \div\left(\alpha_{j}+\alpha_{3}\right)\left(1-e^{-\left(\frac{e^{x_{j}}}{\lambda}\right)^{k}}\right)^{\alpha_{3}} \\
& f_{X_{i} \mid X_{j}}^{(2)}\left(x_{i} \mid x_{j}\right)=\alpha_{i} k\left(\frac{e^{x_{i}}}{\lambda}\right)^{k} e^{-\left(\frac{e^{x_{i}}}{\lambda}\right)^{k}}\left(1-e^{-\left(\frac{e^{x_{i}}}{\lambda}\right)^{k}}\right)^{\alpha_{i}-1} \\
& f_{X_{i} \mid X_{j}}^{(3)}\left(x_{i} \mid x_{j}\right)=\frac{\alpha_{3}}{\alpha_{i}+\alpha_{3}}\left(1-e^{-\left(\frac{e^{x_{i}}}{\lambda}\right)^{k}}\right)^{\alpha_{i}} .
\end{aligned}
$$

PROOF. The proof follows immediately by substituting the joint probability density function of $\left(X_{1}, X_{2}\right)$ given in $15,(16)$ and (17) and the marginal probability density function of $X_{i}(i=1,2)$ given in 20], using the relation

$$
f_{X_{i} \mid X_{j}}\left(x_{i} \mid x_{j}\right)=\frac{f_{X_{i}, X_{j}}\left(x_{i}, x_{j}\right)}{f_{X_{j}}\left(x_{j}\right)}, i=1,2 .
$$

\section{MAXIMUM LIKELIHOOD ESTIMATORS OF BNGEVD}

Kundu and Gupta [1] used the method of maximum likelihood to estimate the unknown parameters of the bivariate generalized exponential distribution. In the same way we use the method of maximum likelihood to estimate the unknown parameters of the BNGVD distribution.

Suppose $\left(\left(x_{11}, x_{21}\right), \ldots,\left(x_{1 n}, x_{2 n}\right)\right)$ is a random sample from BNGVD distribution.Consider the following notation

$I_{1}=\left\{i ; x_{1 i}<x_{2 i}\right\}, \quad I_{2}=\left\{i ; x_{1 i}>x_{2 i}\right\}, \quad I_{3}=$ $\left\{i ; x_{1 i}=x_{2 i}=x_{i}\right\}, I=I_{1} \cup I_{2} \cup I_{3},\left|I_{1}\right|=n_{1},\left|I_{2}\right|=n_{2}$, $\left|I_{3}\right|=n_{3}$, and $n_{1}+n_{2}+n_{3}=n$.

The likelihood function of the sample of size $n$ is given by:

$l\left(\alpha_{1}, \alpha_{2}, \alpha_{3}, \lambda, k\right)={ }_{i=1}^{n_{1}} f_{1}\left(x_{1 i}, x_{2 i}\right)_{i=1}^{n_{2}} f_{2}\left(x_{1 i}, x_{2 i}\right)_{i=1}^{n_{3}} f_{3}\left(x_{i}\right)$ The log-likelihood function can be expressed as $L\left(\alpha_{1}, \alpha_{2}, \alpha_{3}, \lambda, k\right)=\ln l\left(\alpha_{1}, \alpha_{2}, \alpha_{3}, \lambda, k\right)$

$=n_{1} \ln \left(\alpha_{1}+\alpha_{3}\right)+n_{1} \ln (k)+k \sum_{i=1}^{n_{1}} \ln \left(\frac{e^{x_{1 i}}}{\lambda}\right)-$ $\sum_{i=1}^{n_{1}}\left(\frac{e^{x_{1 i}}}{\lambda}\right)^{k}+\left(\alpha_{1}+\alpha_{3}-1\right) \sum_{i=1}^{n_{1}} \ln \left(1-e^{-\left(\frac{e^{x_{1 i}}}{\lambda}\right)^{k}}\right)+$ $n_{1} \ln \left(\alpha_{2}\right)+n_{1} \ln (k)+k \sum_{i=1}^{n_{1}} \ln \left(\frac{e^{x_{2 i}}}{\lambda}\right)-\sum_{i=1}^{n_{1}}\left(\frac{e^{x_{2 i}}}{\lambda}\right)^{k}+$ $\left(\alpha_{2}-1\right) \sum_{i=1}^{n_{1}} \ln \left(1-e^{-\left(\frac{e^{x_{2 i}}}{\lambda}\right)^{k}}\right)+n_{2} \ln \left(\alpha_{1}\right)+$ $n_{2} \ln (k)+k \sum_{i=1}^{n_{2}} \ln \left(\frac{e^{x_{1 i}}}{\lambda}\right)-\sum_{i=1}^{n_{2}}\left(\frac{e^{x_{1 i}}}{\lambda}\right)^{k}+$ $\left(\alpha_{1}-1\right) \sum_{i=1}^{n_{2}} \ln \left(1-e^{-\left(\frac{e^{x_{1 i}}}{\lambda}\right)^{k}}\right)+n_{2} \ln \left(\alpha_{2}+\alpha_{3}\right)+$ $n_{2} \ln (k)+k \sum_{i=1}^{n_{2}} \ln \left(\frac{e^{x_{2 i}}}{\lambda}\right) \quad-\quad \sum_{i=1}^{n_{2}}\left(\frac{e^{x_{2 i}}}{\lambda}\right)^{k}+$ $\left(\alpha_{2}+\alpha_{3}-1\right) \sum_{i=1}^{n_{2}} \ln \left(1-e^{-\left(\frac{e^{x_{2 i}}}{\lambda}\right)^{k}}\right)+n_{3} \ln \left(\alpha_{3}\right)$

$+n_{3} \ln (k)+k \sum_{i=1}^{n_{3}} \ln \left(\frac{e^{x_{i}}}{\lambda}\right)-\sum_{i=1}^{n_{3}}\left(\frac{e^{x_{i}}}{\lambda}\right)^{k}+$ $\left(\alpha_{1}+\alpha_{2}+\alpha_{3}-1\right) \sum_{i=1}^{n_{3}} \ln \left(1-e^{-\left(\frac{e^{x_{i}}}{\lambda}\right)^{k}}\right)$ 
Differentiating the log-likelihood with respect to $\alpha_{1}, \alpha_{2}, \alpha_{3}, \lambda$ and $k$ respectively, and setting the results equal to zero, we have

$$
\begin{aligned}
& \frac{\partial L}{\partial \alpha_{1}}=\frac{n_{1}}{\alpha_{1}+\alpha_{3}}+\sum_{i=1}^{n_{1}} \ln \left(1-e^{-\left(\frac{e^{x_{1 i}}}{\lambda}\right)^{k}}\right)+\frac{n_{2}}{\alpha_{1}} \\
& +\sum_{i=1}^{n_{2}} \ln \left(1-e^{-\left(\frac{e^{x_{11}}}{\lambda}\right)^{k}}\right)+\sum_{i=1}^{n_{3}} \ln \left(1-e^{-\left(\frac{e^{x_{i}}}{\lambda}\right)^{k}}\right) \\
& \frac{\partial L}{\partial \alpha_{2}}=\frac{n_{1}}{\alpha_{2}}+\sum_{i=1}^{n_{1}} \ln \left(1-e^{-\left(\frac{e^{x_{2 i}}}{\lambda}\right)^{k}}\right)+\frac{n_{2}}{\alpha_{2}+\alpha_{3}} \\
& \quad+\sum_{i=1}^{n_{2}} \ln \left(1-e^{-\left(\frac{e^{x_{2 i}}}{\lambda}\right)^{k}}\right)+\sum_{i=1}^{n_{3}} \ln \left(1-e^{-\left(\frac{e^{x_{i}}}{\lambda}\right)^{k}}\right) \\
& \frac{\partial L}{\partial \alpha_{3}}=\frac{n_{1}}{\alpha_{1}+\alpha_{3}}+\sum_{i=1}^{n_{1}} \ln \left(1-e^{-\left(\frac{e^{x} 1 i}{\lambda}\right)^{k}}\right)+\frac{n_{2}}{\alpha_{2}+\alpha_{3}}+\frac{n_{3}}{\alpha_{3}} \\
& \quad+\sum_{i=1}^{n_{2}} \ln \left(1-e^{-\left(\frac{e^{x_{2} i}}{\lambda}\right)^{k}}\right)+\sum_{i=1}^{n_{3}} \ln \left(1-e^{-\left(\frac{e^{x_{i}}}{\lambda}\right)^{k}}\right)
\end{aligned}
$$

$$
\begin{aligned}
& \frac{\partial L}{\partial k} \quad=\quad \frac{n_{1}}{k}+\sum_{i=1}^{n_{1}} \ln \left(\frac{e^{x} 1 i}{\lambda}\right)+ \\
& \left(\alpha_{1}+\alpha_{3}-1\right) \sum_{i=1}^{n_{1}} \frac{e^{-\left(\frac{e^{x_{1 i}}}{\lambda}\right)^{k}}\left(\frac{e^{x_{1 i}}}{\lambda}\right)^{k} \ln \left(\frac{e^{x_{1 i}}}{\lambda}\right)}{1-e^{-\left(\frac{e^{x} x_{i}}{\lambda}\right)^{k}}}+\frac{n_{1}}{k}+ \\
& \sum_{i=1}^{n_{1}} \ln \left(\frac{e^{x_{2 i}}}{\lambda}\right)+\left(\alpha_{2}-1\right) \sum_{i=1}^{n_{1}} \frac{e^{-\left(\frac{e^{x_{2 i}}}{\lambda}\right)^{k}}\left(\frac{e^{x_{2 i}}}{\lambda}\right)^{k} \ln \left(\frac{e^{x_{2 i}}}{\lambda}\right)}{1-e^{-\left(\frac{e^{x} x_{2 i}}{\lambda}\right)^{k}}}+ \\
& \frac{n_{2}}{k}+\sum_{i=1}^{n_{2}} \ln \left(\frac{e^{x_{1 i}}}{\lambda}\right)+\sum^{n_{3}}+\frac{n_{2}}{k}+
\end{aligned}
$$

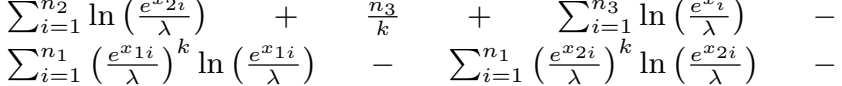

$$
\begin{aligned}
& \sum_{i=1}^{n_{2}}\left(\frac{e^{x_{1 i}}}{\lambda}\right)^{k} \ln \left(\frac{e^{x_{1 i}}}{\lambda}\right) \quad-\quad \sum_{i=1}^{n_{2}}\left(\frac{e^{x_{2 i}}}{\lambda}\right)^{k} \ln \left(\frac{e^{x_{2 i}}}{\lambda}\right) \quad+ \\
& \left(\alpha_{1}-1\right) \sum_{i=1}^{n_{2}} \frac{e^{-\left(\frac{e^{x_{1 i}}}{\lambda}\right)^{k}}\left(\frac{e^{x_{1 i}}}{\lambda}\right)^{k} \ln \left(\frac{e^{x_{1 i}}}{\lambda}\right)}{1-e^{-\left(\frac{e^{x} x_{i i}}{\lambda}\right)^{k}}} \\
& \left(\alpha_{2}+\alpha_{3}-1\right) \sum_{i=1}^{n_{2}} \frac{e^{-\left(\frac{e^{x_{2 i}}}{\lambda}\right)^{k}}\left(\frac{e^{x_{2 i}}}{\lambda}\right)^{k} \ln \left(\frac{e^{x_{2 i}}}{\lambda}\right)}{1-e^{-\left(\frac{e^{x} 2 i}{\lambda}\right)^{k}}} \\
& \left(\alpha_{1}+\alpha_{2}+\alpha_{3}-1\right) \sum_{i=1}^{n_{3}} \frac{e^{-\left(\frac{e^{x_{i}}}{\lambda}\right)^{k}}\left(\frac{e^{x_{i}}}{\lambda}\right)^{k} \ln \left(\frac{e^{x_{i}}}{\lambda}\right)}{1-e^{-\left(\frac{e^{x_{i}}}{\lambda}\right)^{k}}} \\
& -\sum_{i=1}^{n_{3}}\left(\frac{e^{x_{i}}}{\lambda}\right)^{k} \ln \left(\frac{e^{x_{i}}}{\lambda}\right)
\end{aligned}
$$

$$
\begin{aligned}
& \frac{\partial L}{\partial \lambda} \quad=\frac{-n_{1} k}{\lambda}+\frac{k}{\lambda} \sum_{i=1}^{n_{1}}\left(\frac{e^{x_{1 i}}}{\lambda}\right)^{k}- \\
& \left(\alpha_{1}+\alpha_{3}-1\right) \sum_{i=1}^{n_{1}} \frac{\frac{k}{\lambda}\left(\frac{e^{x_{1 i}}}{\lambda}\right)^{k} e^{-\left(\frac{e^{x_{1 i}}}{\lambda}\right)^{k}}}{1-e^{-\left(\frac{e^{x} 1 i}{\lambda}\right)^{k}}}-\frac{n_{1} k}{\lambda}+
\end{aligned}
$$

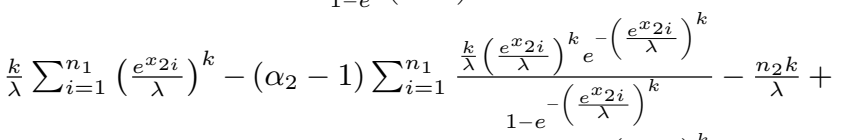

$$
\begin{aligned}
& \frac{k}{\lambda} \sum_{i=1}^{n_{2}}\left(\frac{e^{x_{1 i}}}{\lambda}\right)^{k}+\left(\alpha_{1}-1\right) \sum_{i=1}^{n_{2}} \frac{\frac{k}{\lambda}\left(\frac{e^{x_{1 i}}}{\lambda}\right)^{k} e^{-\left(\frac{e^{x_{1 i}}}{\lambda}\right)^{k}}}{1-e^{-\left(\frac{e^{x_{1 i}}}{\lambda}\right)^{k}}}-\frac{n_{2} k}{\lambda}+
\end{aligned}
$$

$$
\begin{aligned}
& \frac{k}{\lambda} \sum_{i=1}^{n_{2}}\left(\frac{e^{x_{2 i}}}{\lambda}\right)^{k}-\left(\alpha_{2}+\alpha_{3}-1\right) \sum_{i=1}^{n_{2}} \frac{\frac{k}{\lambda}\left(\frac{e^{x_{2 i}}}{\lambda}\right)^{k} e^{-\left(\frac{e^{x_{2 i}}}{\lambda}\right)^{k}}}{1-e^{-\left(\frac{e^{x_{2 i}}}{\lambda}\right)^{k}}}- \\
& \frac{n_{3} k}{\lambda}+\frac{k}{\lambda} \sum_{i=1}^{n_{3}}\left(\frac{e^{x_{i}}}{\lambda}\right)^{k} \\
& \quad-\left(\alpha_{1}+\alpha_{2}+\alpha_{3}-1\right) \sum_{i=1}^{n_{3}} \frac{\frac{k}{\lambda}\left(\frac{e^{x_{i}}}{\lambda}\right)^{k} e^{-\left(\frac{e^{x_{i}}}{\lambda}\right)^{k}}}{1-e^{-\left(\frac{e^{x_{i}}}{\lambda}\right)^{k}}}
\end{aligned}
$$

\begin{tabular}{|c|c|c|c|c|c|c|c|}
\hline \multicolumn{8}{|c|}{ Table 4 UEFA Champion's League Data } \\
\hline$X_{1}$ & $X_{2}$ & $X_{1}$ & $X_{2}$ & $X_{1}$ & $X_{2}$ & $X_{1}$ & $X_{2}$ \\
\hline 26 & 20 & 66 & 62 & 51 & 28 & 42 & 3 \\
\hline 63 & 18 & 25 & 9 & 76 & 64 & 27 & 47 \\
\hline 19 & 19 & 41 & 3 & 64 & 15 & 28 & 28 \\
\hline 66 & 85 & 16 & 75 & 26 & 48 & 2 & 2 \\
\hline 40 & 40 & 18 & 18 & 16 & 16 & & \\
\hline 49 & 49 & 22 & 14 & 44 & 13 & & \\
\hline 8 & 8 & 42 & 42 & 25 & 14 & & \\
\hline 69 & 71 & 36 & 52 & 55 & 11 & & \\
\hline 39 & 39 & 34 & 34 & 49 & 49 & & \\
\hline 82 & 48 & 53 & 39 & 24 & 24 & & \\
\hline 72 & 72 & 54 & 7 & 44 & 30 & & \\
\hline
\end{tabular}

The maximum likelihood estimates $\widehat{\alpha}_{1}, \widehat{\alpha}_{2}, \widehat{\alpha}_{3}, \widehat{\lambda}$ and $\widehat{k}$ of the unknown parameters $\alpha_{1}, \alpha_{2}, \alpha_{3}, \lambda$ and $k$ respectively, are obtained by solving Equations 22, - 26.

\section{DATA ANALYSIS OF BNGEVD}

In this section, a real data set is used to compare the goodness of

\begin{tabular}{|c|c|}
\hline Model & MLEs \\
\hline MO & $\begin{array}{c}\widehat{\lambda}_{1}=0.012, \widehat{\lambda}_{2}=0.014 \\
\widehat{\lambda}_{3}=0.022\end{array}$ \\
\hline BNGEV & $\begin{array}{c}\widehat{\alpha}_{1}=3.682, \widehat{\alpha}_{2}=1.398 \\
\widehat{\alpha}_{3}=3.386, \widehat{\lambda}=57.447 \\
\widehat{k}=0.022\end{array}$ \\
\hline
\end{tabular}
fiting of the Marshall-Olkin bivariate exponential (MO) distribution and Bivariate New Generalized Extreme value (BNGEV) distribution. The data set (see Table 4) was first analyzed in [1] and represents the soccer data where at least one goal is scored by the home team and at least one goal is scored directly from apenalty kick, foul kick or any other direct kick( all of them will be called kick goals) by any team that has been considered. It is a bivariate data set, and the variables $X_{1}$ and $X_{2}$ are as follows: $X_{1}$ represents the time in minutes of the first kick goal scored by any team and $X_{2}$ represents the first goal of any type scored by the home team. Clearly, the variables $X_{1}$ and $X_{2}$ have the following structure: (i) $X_{1}<X_{2}$, (ii) $X_{1}=X_{2}$, (iii) $X_{1}>X_{2}$.

The required numerical evaluations are carried out using the Package of Mathcad software. Table 5 provides the MLEs of the model parameters. The model selection is carried out using the $A I C$ (Akaike information criterion) and the $B I C$ (Bayesian information criterion). 


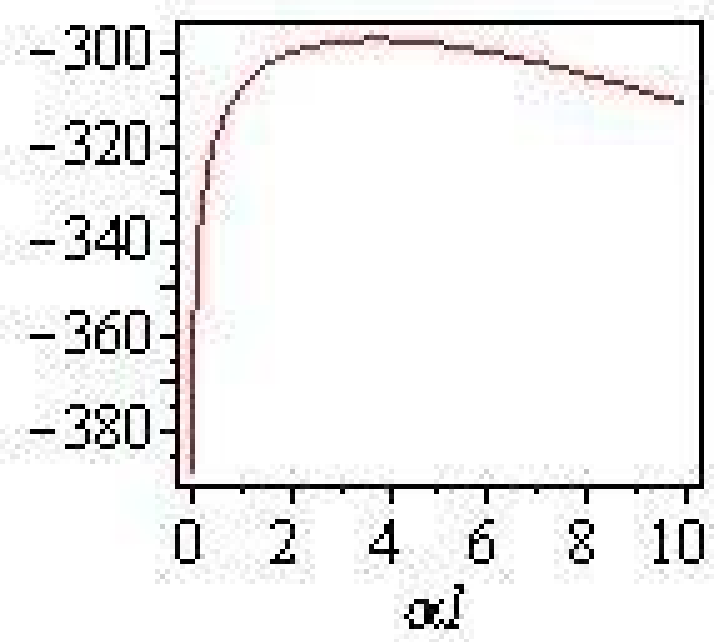

Fig. 6. The profile of the log-likelihood function of $\alpha_{1}$

Table 6: The values of $L, A I C$ and $B I C$.
\begin{tabular}{|c|c||c||c|}
\hline \hline Model & $L$ & $A I C$ & $B I C$ \\
\hline \hline MO & -339.006 & 684.012 & -344.423 \\
& & & \\
\hline BNGEV & -297.342 & 604.684 & -306.369 \\
& & & \\
\hline
\end{tabular}

Since the values of $-L, A I C$ and $B I C$ (see Table 6) are smaller for the BNGEV distribution compared with those values of the other models, then the new distribution seems to be a very competitive model to these data

The profiles of the log-likelihood function of $\alpha_{1}, \alpha_{2}, \alpha_{3}, \lambda$ and $k$ of BNGEV for UEFA Champion's League data are ploted in Fig. 5, Fig. 6 and Fig. 7. From the plots of the profiles of the log-likelihood function of $\alpha_{1}, \alpha_{2}, \alpha_{3}, \lambda$ and $k$,we observe that the likelihood equations have a unique solution.

\section{CONCLUSIONS}

In this paper, we proposed a new generalized extreme value (NGEV) distribution. Some statistical properties of this distribution have been studied and discussed such as quantile, median, moment generating function and moments of order statistics. The maximum likelihood estimators of the parameters are derived. A real data set is analyzed using the new distribution, Gumbel type2 (G type-2) distribution, Exponentiated Fréchet (EF) distribution, Fréchet distribution and lognormal (LN) distribution. Baised on the comparisons between all these models, we conclude that, the introduced model is highly competitive in the sense of fitting this real data set. Also, bivariate New Generalized Extreme Value (BNGEV) distribution.is introduced. Marginal and conditional distribution functions are studied. Furthermore, maximum likelihood estimates (MLEs) of the parameters are presented. A real data set is analyzed using the new distribution and Marshall-Olkin bivariate exponential (MO) distribution. Baised on the comparisons between all these models, we conclude that, the introduced model is highly competitive in the sense of fitting this real data set.

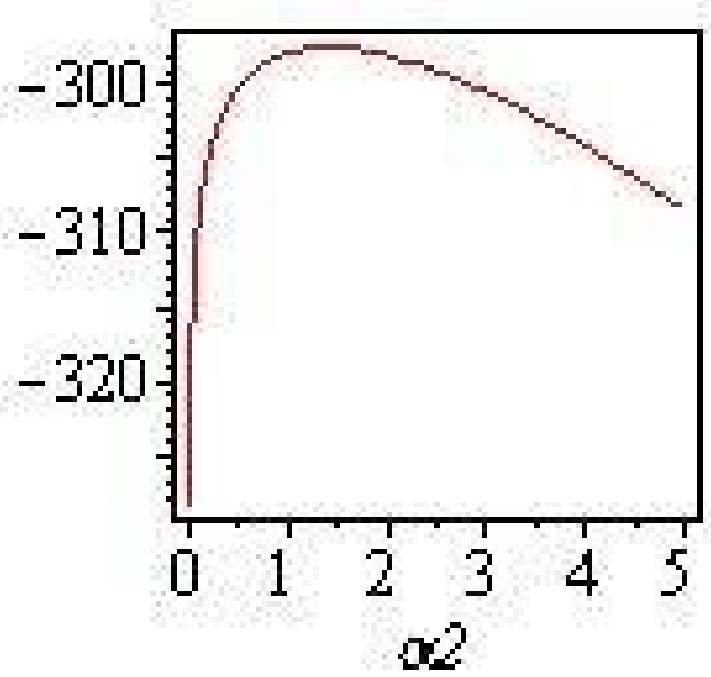

Fig. 7. The profile of the log-likelihood function of $\alpha_{2}$

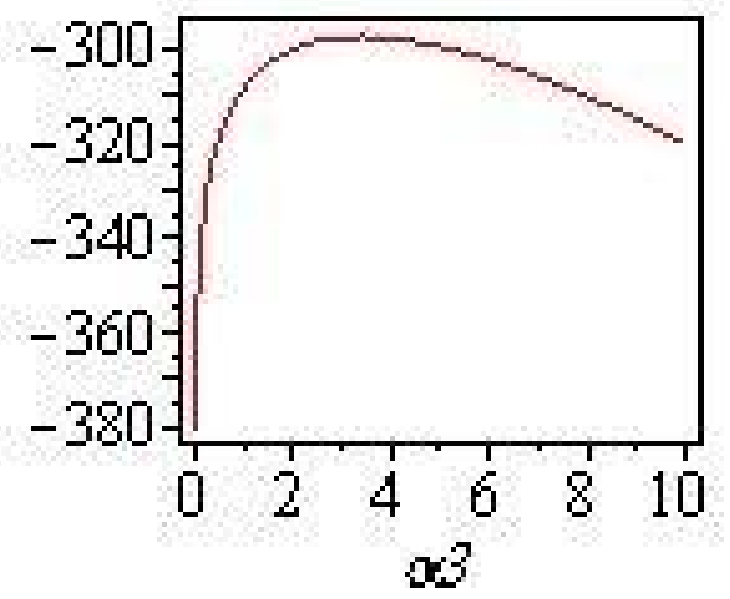

Fig. 8. The profile of the log-likelihood function of $\alpha_{3}$

\section{REFERENCES}

[1] Kundu, D. and Gupta, R. D. (2009). Bivariate generalized exponential distribution. Journal of Multivariate Analysis, vol. 100, no. 4, 581-593.

[2] Marshall, A. W. Olkin, I. A. (1967). A multivariate exponential distribution. Journal of the American Statistical Association. $62,30-44$.

[3] Sarhan, A. and Balakrishnan, N. (2007). A new class of bivariate distributions and its mixture. Journal of the Multivariate Analysis. 98, 1508-1527.

[4] El-Bassiouny, A. H., El-Damcese, M., Mustafa, A., and Eliwa, M. (2015). Bivariate exponentaited generalized WeibullGompertz distribution. arXiv preprint arXiv:1501.02241.

[5] El-Gohary, A., El-Bassiouny, A. H. and El-Morshedy, M. (2016). Bivariate exponentiated modified Weibull extension 


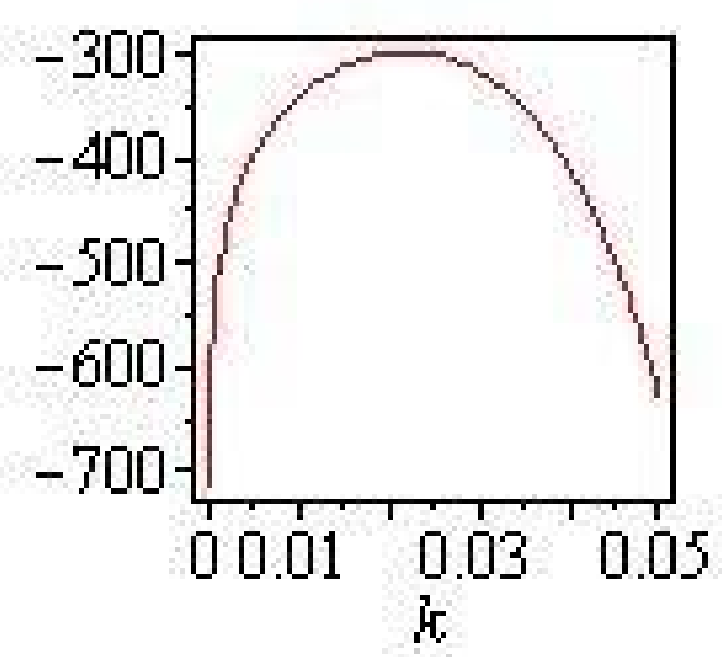

Fig. 9. The profile of the log-likelihood function of $k$

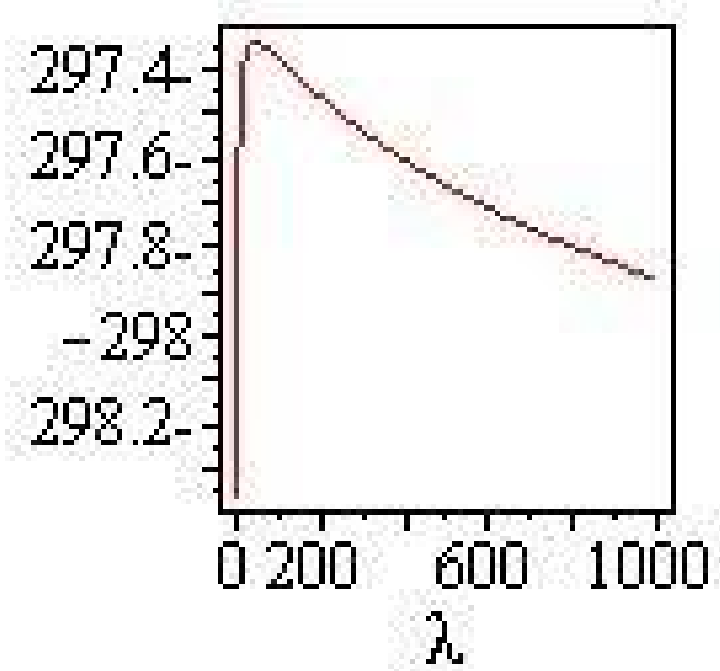

Fig. 10. The profile of the log-likelihood function of $\lambda$

distribution. Journal of Statistics Applications \& Probability 5, No. 1, 1-12.

[6] Ammar M. Sarhan, David C. Hamilton, Bruce Smith and Debasis Kundu. (2011). The bivariate generalized linear failure rate distribution and its multivariate extension. Computational Statistics and Data Analysis 55, 644-654.

[7] A. Z. Afify, Z. M. Nofal, and N. S. Butt, "Transmuted complementary weibull geometric distribution," Pakistan Journal of Statistics and Operation Research, vol. 10, no. 4, pp. 435-454, 2014.

[8] Meintanis, S. G. (2007). Test of fit for Marshall-Olkin distributions with applications. Journal of Statistical Planning and Inference 137,3954-3963.
[9] Mudholkar GS and Srivastava DK (1993). Exponentiated Weibull family for analyzing bathtub failure-rate data, IEEE Transactions on Reliability, 42, 299-302. 